\title{
Why You Think Milan is Larger than Modena: Neural Correlates of the Recognition Heuristic
}

\author{
Kirsten G. Volz ${ }^{1}$, Lael J. Schooler ${ }^{2}$, Ricarda I. Schubotz ${ }^{1}$, \\ Markus Raab ${ }^{3}$, Gerd Gigerenzer ${ }^{2}$, and D. Yves von Cramon ${ }^{1}$
}

\begin{abstract}
When ranking two alternatives by some criteria and only one of the alternatives is recognized, participants overwhelmingly adopt the strategy, termed the recognition heuristic $(\mathrm{RH})$, of choosing the recognized alternative. Understanding the neural correlates underlying decisions that follow the RH could help determine whether people make judgments about the RH's applicability or simply choose the recognized alternative. We measured brain activity by using functional magnetic resonance

imaging while participants indicated which of two cities they
\end{abstract}

thought was larger (Experiment 1) or which city they recognized (Experiment 2). In Experiment 1, increased activation was observed within the anterior frontomedian cortex (aFMC), precuneus, and retrosplenial cortex when participants followed the $\mathrm{RH}$ compared to when they did not. Experiment 2 revealed that $\mathrm{RH}$ decisional processes cannot be reduced to recognition memory processes. As the aFMC has previously been associated with selfreferential judgments, we conclude that $\mathrm{RH}$ decisional processes involve an assessment about the applicability of the RH.

\section{INTRODUCTION}

We often need to rank two alternatives by some criterion, for example, which road to a city is faster. In one experiment done to explore how people do such rankings, Goldstein and Gigerenzer (2002) presented U.S. students with pairs of large U.S. cities and with pairs of large German cities. The task was to infer which city in each pair had the larger population. The students performed equally well on the German and U.S. city pairs. How can this be that the students performed as well with German cities as with the American cities, when they knew almost nothing about the German cities? Goldstein and Gigerenzer found that people reliably employ a remarkably effective decision strategy they dubbed the recognition beuristic $(\mathrm{RH})$, which can be stated as follows: "If one of two objects is recognized and the other is not, then infer that the recognized object has the higher value with respect to the criterion" (p. 76). According to Goldstein and Gigerenzer, the students' imperfect recognition of the German cities allowed them to frequently apply the $\mathrm{RH}$, which led them to pick the recognized German cities, which tend to be larger than those that are unrecognized. The students could not use this heuristic when comparing U.S. cities, though, because they recognized all of them.

The RH works only in domains, or environments, in which recognition is correlated-in either direction-

\footnotetext{
${ }^{1}$ Max Planck Institute for Human Cognitive and Brain Sciences, Germany, ${ }^{2}$ Max Planck Institute for Human Development, Germany, ${ }^{3}$ University of Flensburg, Germany
}

with the inaccessible criterion being predicted, for example, between city name recognition and population size. To the extent that the RH functions well by exploiting characteristics of an environment, it is ecologically rational with respect to that particular environment. Goldstein and Gigerenzer (2002) argue the effectiveness of the RH in the city judgment task depends on three correlations: Population size is reflected by how often a city is mentioned in the media, which in turn influences the probability of recognition (Figure 1). Thus, recognition information correlates with and can be used to infer the inaccessible criterion. Accordingly, the effective use of the RH depends on the outcome of two processes. The first yields whether the alternatives are recognized and so whether the $\mathrm{RH}$ can be applied. The second process assesses whether the RH should be applied, and so is essentially a judgment about its ecological rationality. The judgment of ecological rationality could, for example, depend on an assessment of whether the chain of correlations linking the criterion to recognition through the mediator (e.g., the frequency of city name in the media) justifies following the RH.

Yet, it is not necessarily the case that subjects have to judge the ecological rationality of the RH before applying it. Instead, subjects could simply choose the recognized alternative without considering why recognition should be predictive of population size. Such a strategy would be successful here because recognition is so highly correlated with how often a stimulus occurs in the environment. 


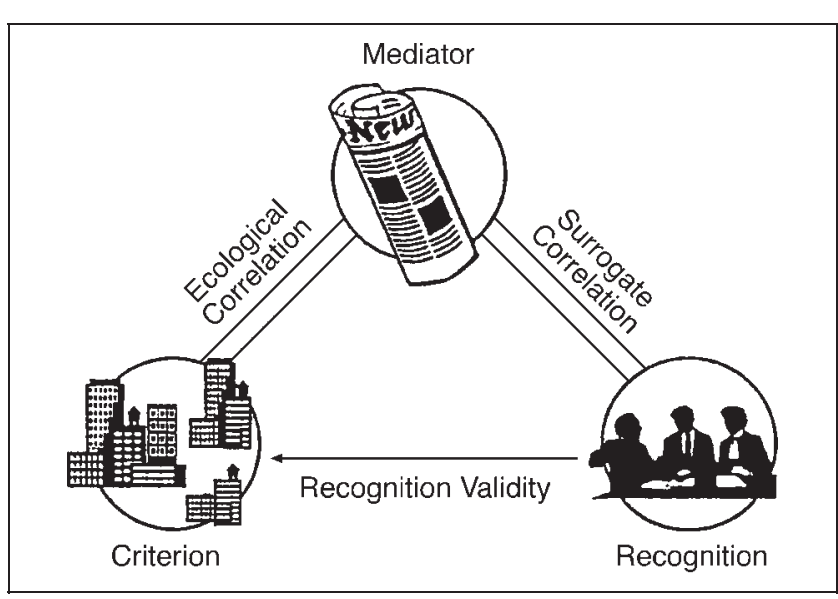

Figure 1. The ecological rationality of the recognition heuristic $(\mathrm{RH})$, that is, the ability to exploit the structure of the information in the environment: An inaccessible criterion (e.g., the population size of a certain city) is reflected but not revealed by a mediator variable (e.g., the number of times a city is mentioned in the media), and the mediator influences the probability of recognition. The recognition information is then used to infer the criterion. This inference is successful only if recognition is correlated with the criterion. Adapted from Goldstein and Gigerenzer (2002).

Understanding the neural correlates underlying the use of the RH could help determine whether RH-based decisional processes depend on additional judgments of ecological rationality. Such judgments of ecological rationality should draw on brain areas beyond those known to reflect recognition memory processes.

Some have concluded from behavioral studies that declarative memory (episodic memory [events] and semantic memory [facts]; cf. Squire, 1994) is supported by at least two distinct processes: The assessment of what has been termed item familiarity and the recollection of the context in which an item was encountered before (Yonelinas et al., 2002). Neuropsychological as well as neuroscientific evidence support the proposed dual-process framework in that recollection and familiarity draw on distinct neural mechanisms: Whereas the hippocampus is considered to play a crucial role in recollection (e.g., Aggleton et al., 2005; Ranganath et al., 2003; Yonelinas et al., 2002; Aggleton \& Shaw, 1996; for an overview, see Rugg \& Yonelinas, 2003), adjacent medial temporal lobe (MTL) regions (perirhinal cortex) are hypothesized to reflect familiarity-based recognition (Yonelinas et al., 2002). Yet, a recent review on the MTL outlines that the available data may not support such a simple dichotomy (Squire, Stark, \& Clark, 2004). Rather, the authors suggest that the various anatomical components of the MTL all signal information that is significant to successful recognition memory performance: The hippocampal formation provides a conjunctive recognition signal, whereas adjacent cortices provide stimulus-specific (recognition) signals. Both components are considered necessary for intact recognition memory performance and hence may account for why there is only little support of a sharp distinction of labor within the MTL. Besides these cortices, parietal areas have consistently been shown to be crucial for remembering, particularly for episodic retrieval processes (Wagner, Shannon, Kahn, \& Buckner, 2005). For example, (lateral and medial) parietal areas have been shown to increase their activity to recognized old items as well as to erroneously recognized new items and to correlate with the subjective experience of remembering. Accordingly, if RH-based decisional processes in the present study were not only premised on recognition information but rather depend on additional judgments of ecological rationality, we expected regions beyond MTL regions and parietal areas to be activated when participants decided according to the RH.

Thus, by using functional magnetic resonance imaging (fMRI) and an adapted version of the two-alternative forced-choice task used by Goldstein and Gigerenzer (2002), we contrasted the neural correlates of decisions based on the RH to those in which the heuristic was not applied. Yet, finding activation in areas besides those regions known to reflect recognition memory processes would not definitively rule out an alternative interpretation, namely, that those areas reflect decisional processes that simply opt for recognized alternatives. For this reason, we ran the same experiment again with a different group of participants and with the instruction to simply choose the recognized city in each pair (Experiment 2). The test of city recognition and the paired comparison test were run as a between-subjects design to avoid potential carryover effects. In Experiment 2, participants were asked to execute the task "Which city do you know?" instead of "Which city is larger?" By contrasting trials in which participants were instructed to choose the recognized city with trials in which they were instructed to choose the unrecognized city, we built a contrast parallel to the RH contrast from Experiment 1. In doing so, we could compare the activation patterns of both experiments. To the extent that the RH-based decisional processes (1) can be adequately explained by participants simply choosing the recognized alternative and (2) do not depend on a judgment of the ecological rationality, the activation patterns of the two contrasts should not differ with respect to the critical activations.

\section{METHODS}

\section{Experiment 1}

\section{Participants}

Eighteen (10 women, mean age 25.6 years, SD 3.4, range 20-32 years) right-handed, healthy volunteers participated in the fMRI experiment. Informed consent was obtained prior to the experiment from each participant according to the Declaration of Helsinki. Experimental standards were approved by the local ethics 
committee of the University of Leipzig. Data were handled anonymously.

\section{Stimuli, Task, and Experimental Session}

Stimuli consisted of two concurrently presented words, one on the right and one on the left side of the screen (horizontal visual angle, $11^{\circ}$; vertical visual angle, $1.7^{\circ}$ ). Participants had their index fingers on a left and a right response button, spatially corresponding to the stimulus locations on the screen. Within each trial a cue was presented for $500 \mathrm{msec}$ signaling that the next trial was about to start, followed by a fixation cross presented for 500 msec. Subsequently, word pairs were presented for a maximum of 4 sec during which participants' response was recorded. As soon as participants indicated their choice by a button press, the stimuli disappeared and were replaced by a fixation cross. No performance feedback was delivered whatsoever. The participants' task was to indicate which city in each pair had the larger population.

An experimental session consisted of 140 experimental trials and 30 null events, in which no stimulus was presented and so the blood oxygenation level dependent (BOLD) response was allowed to return to a baseline state. All trials lasted for 8 sec each (i.e., four scans of repetition time [TR] 2 sec). To allow for measurements to be taken at numerous time points along the BOLD signal curve, the onset of each stimulus presentation relative to the beginning of the first of the four scans was varied randomly in four time steps (0, 500,1000 , and $1500 \mathrm{msec}$ ). The purpose of this procedure was to enhance the temporal resolution of the image acquisition (Birn, Cox, \& Bandettini, 2002; Miezin, Maccotta, Ollinger, Petersen, \& Buckner, 2000).

After the fMRI session, a recognition test was administered in which participants had to indicate whether or not they knew each city from before the experimental session. It was emphasized to the participants that they should declare as recognized only those cities that they had heard of before the functional session. These data were used to determine individual trial types, that is, whether both, none, or only one of the cities were recognized. Accordingly, we could individually determine RR (recognized-recognized), RU (recognizedunrecognized), and UU (unrecognized-unrecognized) trials. Furthermore, the data of the recognition test were also used to assess in which RU trials participants decided in favor of the recognized city, that is, the application of the RH. After the recognition test, participants were asked to fill out a questionnaire asking wherefrom they knew most of the cities; subsequently, they were debriefed and thanked.

Our experimental design called for the city comparison city task to always come first, followed by the recognition test, rather than counterbalancing the task order. Having the recognition test before the city task could have biased participants towards using the $\mathrm{RH}$ by making salient to the participants that we (the experimenters) were interested in whether or not they recognized the stimuli. In addition, by keeping a fixed task order, we were able to reliably measure the hemodynamic response elicited by recognition judgments that are presumed to underlie the RH. That is, judgments about whether the city can be recognized from their daily life, and so can be used to make inferences about the cities. Had the recognition task come first, the participants would have had to judge not only whether they recognized the city, but also whether the source of the recognition was just from the experiment or possibly from elsewhere. The additional demands of this discrimination task means that the recognition judgments from the two task orders could draw on somewhat different brain structures and so involve brain structures that would not otherwise be involved in the application of the RH. Nevertheless, we expect that in the recognition task, participants could reliably report whether they saw the city from the experiment or from elsewhere. That is, they would rarely miscategorize as recognized a city that they had only encountered in the experiment. Support for this come from the studies of Pohl (2006) and Goldstein and Gigerenzer (2002). Neither study found any difference in the recognition rates that depended on the order of the two tasks.

\section{Pilot Study for Stimulus Material Preparation}

A key design goal of the present study was that each city in the experiment should only be seen once, so that participant's recognition judgments would not be contaminated by their experience within the experiment. For this reason, the city task always preceded the recognition test. To construct the trials so that subjects would be faced with approximately equal proportions of RR, RU, and UU trials, we ran a behavioral pilot study to obtain average recognition rates for 400 cities necessary to make 140 experimental trials. In this pilot study, administered as a paper-and-pencil task, 60 students from Berlin and Flensburg were asked to answer the following question: Please indicate if you have heard the name of this city before. Students answered this question for 400 cities from following countries: Argentina, Brazil, Canada, China, France, Great Britain, Holland, India, Iraq, Italy, Japan, Poland, Portugal, Russia, Spain, South Africa, Sweden, Switzerland, and the United States. The cities were blocked by country and both the order of the country blocks and the cities within each country block were randomized. As a complete counterbalancing of the country blocks would result in $20 !\left(=2.4329^{\mathrm{E}+18}\right)$ different orders, to keep things simple, one ordering of stimuli was used for all participants. Countries with a larger number of cities potentially known by a German population had a higher proportion of cities on the list than countries that we expected to be less familiar (e.g., United States 
vs. China). Based on the pilot study England, France, Holland, Italy, Canada, Spain, and the United States were the only countries with approximately equal proportions of recognized and unrecognized cities.

For the paired comparison task a new set of stimuli was generated for each subject as follows. Pairs were generated for each country separately. From those cities that had yet to be included in the experiment, all possible pairs of cities from the same country were generated. For each pair, the expected proportion of participants who would recognize both, just one or neither city was estimated based on the recognition rates of the Flensburg and Berlin participants. Take, for example, the English cities of Sheffield and Poole, with respective recognition rates of .95 and .1, which we refer to, respectively, as $a$ and $b$. By making the strong assumption that these recognition judgments are independent, we can estimate that the probability that the average participant will recognize both cites is $.095(=a \times b)$, that the probability that only one alternative will be recognized is .86 $(=a[1-b]+[1-a] \times b)$ and that the probability that neither city will be recognized is $.045(=[1-a][1-b])$. Next, the city pair that would do the most to equalize the proportion of RR, RU, and UU trials was selected. The process was repeated for the remaining cities.

After the functional session, we compared the recognition rates of all 280 cities between the Berlin and Flensburg participants (60) on the one hand and the Leipzig participants (18) on the other and found recognition values were highly correlated $(r=.91 ; p<.0001)$. Furthermore, the distribution of RR, RU, and UU trials was not significantly different from an equal distribution (Kolmogorov-Smirnov test: $Z=1.10 ; p=.18$ ), as was intended.

\section{Experiment 2}

\section{Participants}

Fourteen (8 women, mean age 25.8 years, SD 2.5, range 22-30 years) right-handed, healthy volunteers participated in the fMRI experiment. Informed consent was obtained prior to the experiment from each participant according to the Declaration of Helsinki. Experimental standards were approved by the local ethics committee of the University of Leipzig. Data were handled anonymously.

\section{Stimuli, Task, and Experimental Session}

Experiment 2 differed from Experiment 1 in the following points: In the functional session, participants had to perform two tasks that were indicated by different color cues; in addition, the city pairs were also presented in the color corresponding to the indicated task. In one task (respond-recognize), participants had to indicate which city they recognized by pressing with their index finger that key spatially corresponding to the stimulus locations on the screen. In those cases when participants recognized both cities, they were instructed to indicate this with their right middle finger, whereas in cases when they recognized none of the cities, they were instructed to indicate this with their left middle finger. The mappings for the middle fingers were counterbalanced between participants. In another task (respondunrecognized) that was signaled by a color cue, participants were required, in case they recognized only one city, to indicate which one they did not recognize. The response had to be made with the index fingers spatially corresponding to the stimulus locations on the screen of the unrecognized city. In case both or none of the cities were recognized, the assignment for the keys stayed the same as in the respond-recognize task, that is, right and left middle fingers. Across the entire experiment, the two tasks were never performed on the same stimuli. Respond-recognize and respond-unrecognized trials were intermixed and presented in random order. One quarter of the trials assigned as RU trials were categorized as such. As in Experiment 1, the actual number of RU trials was individually different depending on the knowledge of the participants. Parallel to Experiment 1, one functional session consisted of 140 experimental trials and 30 null events lasting for 8 sec each. In addition, the procedure of the oversampling was kept identical to that of Experiment 1.

\section{MRI Scanning Procedure}

\section{Imaging}

Imaging was performed on a 3T scanner (Siemens TRIO, Erlangen, Germany). Twenty-two axial slices ( $4 \mathrm{~mm}$ thickness, 20\% spacing, field of view [FOV] $19.2 \mathrm{~cm}$, data matrix of $64 \times 64$ voxels, and in-plane resolution of $3 \mathrm{~mm} \times 3 \mathrm{~mm}$ ) parallel to the bicommissural plane (ACPC) covering the whole brain were acquired using a single-shot echo-planar imaging (EPI) sequence (TR $2 \mathrm{sec}$, echo time [TE] $30 \mathrm{msec}$, flip angle 90 ). One functional run with 872 time points was run with each time point sampling over the 22 slices. Prior to functional runs, 22 anatomical T1-weighted modified driven equilibrium Fourier transform (MDEFT; Norris, 2000; Ugurbil et al., 1993) images (data matrix $256 \times 256$, TR $1.3 \mathrm{sec}$, TE $10 \mathrm{msec}$ ) were acquired as well as 22 T1-weighted EPI images with the same spatial orientation as the functional data. The latter were used to coregister the functional scans with previously acquired high-resolution full-brain 3-D brain scans.

\section{Data Analysis}

The MRI data were processed by using the software package LIPSIA (Lohmann et al., 2001). Functional data were motion-corrected off-line with the Siemens motioncorrection protocol. To correct for the temporal offset 
between the slices acquired in one scan, a cubic spline interpolation was applied. A temporal high-pass filter with a cutoff frequency of $1 / 160 \mathrm{~Hz}$ was used for baseline correction of the signal and a spatial Gaussian filter with $5.65-\mathrm{mm}$ full width half maximum (FWHM) was applied. The anatomical slices were coregistered with the high-resolution full-brain scan that resided in the stereotactic coordinate system and then transformed by linear scaling to a standard size (Talairach \& Tournoux, 1988). The transformation parameters obtained from this step were subsequently applied to the preprocessed functional slices so that the functional slices were also registered into the stereotactic space. This linear normalization process was improved by a subsequent processing step that performed an additional nonlinear normalization known as "demon matching." In this type of nonlinear normalization, an anatomical 3-D data set (i.e., the model) is deformed such that it matches another 3-D anatomical data set (i.e., the source) that serves as a fixed reference image (Thirion, 1998). The voxel size was interpolated during the coregistration from $3 \times 3 \times 4 \mathrm{~mm}$ to $3 \times 3 \times 3 \mathrm{~mm}$. The statistical evaluation was based on a least-squares estimation using the general linear model for serially autocorrelated observations (random effects model; Friston, Frith, Turner, \& Frackowiak, 1995; Worsely \& Friston, 1995). The general linear regression performs a "precoloring" of the data; that is, it applies a temporal Gaussian smoothing with a user-specified kernel width given by the parameter FWHM. The smoothing imposes a temporal autocorrelation that determines the degrees of freedom. An event-related design was implemented; that is, the hemodynamic response function was modeled by means of the experimental conditions for each stimulus (event $=$ onset of stimulus presentation). The design matrix was generated using a synthetic hemodynamic response function and its first and second derivative (Friston et al., 1998) and a response delay of $6 \mathrm{sec}$. The model equation, including the observation data, the design matrix, and the error term, was convolved with a Gaussian kernel of dispersion of 4 sec FWHM to deal with the temporal autocorrelation (Worsley \& Friston,
1995). Contrast images, that is, estimates of the raw score differences between specified conditions, were generated for each subject. The single-subject contrast images entered into a second-level random effects analysis for each of the contrasts. The group analysis consisted of a one-sample $t$ test across the contrast images of all subjects that indicated whether observed differences between conditions were significantly different from zero. Subsequently, $t$ values were transformed into $Z$ scores. Group statistical parametric maps were thresholded at $Z>3.09$ ( $p=.001$, uncorrected). Only clusters of at least six connected voxels (i.e., $162 \mathrm{~mm}^{3}$ ) were reported to ensure an overall imagewise false-positive rate of 5\% (Forman et al., 1995). This nonarbitrary voxel cluster size was determined by using the program AlphaSim (afni. nimh.nih.gov/afni/doc/manual/AlphaSim by Ward, 2000).

\section{RESULTS}

\section{Experiment 1}

\section{Behavioral Results}

Reaction times (RTs) of all RR, RU, and UU trials as well as of correctly answered trials are shown in Table 1 . On average, participants were faced with 55 ( \pm 13 ) RR, 46 $( \pm 5) \mathrm{RU}$, and $39( \pm 15)$ UU trials during an experimental session. A repeated measures analysis of variance of all trials as well as of correctly answered trials revealed the three experimental conditions to differ significantly with regard to RT: [all trials: $F(2,16)=21.93 ; p=.001$; correctly answered trials: $F(2,16)=23.76 ; p=.001$ ] Likewise, the three experimental conditions differed significantly with regard to the rate of correct responses, $F(2,16)=15.83 ; p=.001$. Kolmogorov-Smirnov tests indicate that the means of the subjects' RTs as well as the rate of correct responses were normally distributed (all $Z$ values $>.478$ ). As the standard errors for all conditions were less $2.7 \%$, it is apparent that performance in the RR and RU condition differed significantly from the chance level of $50 \%$, whereas performance in the UU condition was at $50.1 \%$. Regarding the use of the $\mathrm{RH}$, participants applied the heuristic in $84 \%$ of the

Table 1. Behavioral Results of Experiment 1

\begin{tabular}{|c|c|c|c|c|c|c|}
\hline Condition & $R T_{\text {all }}$ & $S E_{\text {(RTall) }}$ & $R T_{\text {corr }}$ & $S E_{(\text {RTcorr })}$ & $\%$ Correct & $S E_{(\% \text { corr })}$ \\
\hline $\mathrm{RR}$ & 2818 & 92 & 2815 & 92 & 63.2 & 1.9 \\
\hline UU & 3024 & 108 & 3074 & 116 & 50.1 & 1.9 \\
\hline RU & 2804 & 89 & 2767 & 84 & 69.2 & 1.5 \\
\hline $\mathrm{RU}_{(\mathrm{R})}$ & 2781 & 90 & 2735 & 80 & 73.5 & 1.5 \\
\hline $\mathrm{RU}_{(\mathrm{U})}$ & 3042 & 116 & 3064 & 159 & 44.2 & 3.8 \\
\hline
\end{tabular}

Reaction times (RTs) of all experimental trials as well as for correctly answered trials and the rates of correct responses (each with standard error $[\mathrm{SE}]$ ) are shown for condition RR (both cities recognized), RU (one city recognized), and UU (no city recognized). The last two rows show RTs and rates of correct responses for RU trials in which participants decided in favor of the recognized alternative $\left(\mathrm{RU}_{(\mathrm{R})}\right)$ and in favor of the unrecognized alternative $\left(\mathrm{RU}_{(\mathrm{U})}\right)$. 
cases. Whether reliance on the RH pays is indicated by the strength of the relationship between recognition and criterion, which is referred to as recognition validity in a specific environment or data set. In the present experiment, the recognition validity averaged .63 , which is calculated as the proportion of times a recognized city was indeed larger than an unrecognized city. Yet, as no performance feedback was given, participants could not determine the recognition validity. Taken together, the present results are consistent with previous results (Goldstein \& Gigerenzer, 2002).

After the experimental session, participants were asked wherefrom they knew most of the cities. It was revealed that more than half of the recognized cities were known from the media, such as newspapers, news on television or the Internet, and novels (63\%). As other important sources, participants indicated schooling (17\%) and holiday (10\%).

\section{MRI Results}

Generally, to control for the RT differences between the conditions (cf. Behavioral Results), we included RT parameters for each condition as covariates. This covariation analysis is capable of removing extraneous variability that derives from RT differences between conditions. To test for the specific neural correlates of RH-based decisions we investigated the hemodynamic response elicited by all RU trials in which the RH was applied as in contrast to all those (RU) trials in which this was not the case (all trials of each condition were included regardless of correctness so as to reliably estimate the beta values). The analysis revealed a significantly higher hemodynamic activity for decisions based on the $\mathrm{RH}$ within the anterior frontomedian cortex (aFMC) and the precuneus bilaterally extending into the retrosplenial cortex (Figure 2, Table 2). No brain region showed significant activation for the inverse contrast. Results remained even if the number of $\mathrm{RH}$ trials was randomly and individually adjusted to match the number of nonRH trials (Figure 2, Table 2). For example, if a participant chose in a specific number of RU trials, say 20 trials, the unrecognized alternative, we would have included in the model 20 randomly chosen RU trials in which the participant selected the recognized alternative.

To investigate which brain areas were involved with successful recognition, RR trials were contrasted with UU trials. Significant activation was revealed bilaterally within the midportion of the parahippocampal gyrus, the ventral striatum extending into the caudate nucleus, the retrosplenial cortex extending into the medial parietal cortex, the intraparietal sulcus, within the posterior frontomedian cortex ( $\mathrm{pFMC}$ ), ventromedial prefrontal cortex (VMPFC), and left inferior prefrontal cortex (LIPFC; Figure 2, Table 2).

To identify regions commonly activated by RH-based decisional processes and recognition memory processes, we calculated a conjunction analysis, that is, a test for a logical AND (Nichols, Brett, Andersson, Wager, \& Poline, 2005). A positive conjunction test implies those regions that are commonly activated across the two kinds of cognitive processes. According to Nichols et al. (2005) a correct test for a logical AND requires that all the comparisons in the conjunction are individually significant at a specified alpha rate. Accordingly, if a voxel showed a $Z$ score less than the critical $Z$ value of 3.09 then the associated voxel in the other $Z$ map was set to zero. That is, if either the map of the RH contrast or the map of the recognition-memory contrast contained a zero, then the conjunction was false. The resulting conjunction map reveals the intersection of the two statistical maps thresholded at a specific alpha rate and hence reliably displays those regions where there is an effect in both maps. In the present study, results of the conjunction analysis revealed significant activation only within two areas: within the retrosplenial cortex and precuneus (Figure 2, Table 2). This finding suggests the retrosplenial cortex activation and precuneus activation but not aFMC activation during RH-based decisions to reflect recognition and retrieval processes.

To test whether medial parietal cortex activation can indeed be considered as an index of the strength of the recognition signal, we calculated a parametric analysis (post hoc) by including a covariate reflecting the average recognition signal per trial, that is, the mean recognition value of the two presented cities. The recognition value for each city in turn was calculated as the percentage of participants in Experiment 1 that recognized it. When testing for the effect of an increasing recognition strength, the same activation pattern as for the recognition memory contrast was revealed: activation revealed bilaterally within the midportion of the parahippocampal gyrus, ventral striatum, retrosplenial cortex extending into the medial parietal cortex, intraparietal sulcus bilaterally, within the pFMC, VMPFC, and LIPFC. Accordingly, medial parietal cortex activation that was revealed when participants applied the RH as compared when they did not is taken to reflect the strength of the recognition signal.

\section{Experiment 2}

\section{Behavioral Results}

Behavioral results of Experiment 2 are shown in Table 3: RT of the four experimental conditions (trials in which participants recognized both cities, neither, or one city and had to choose the recognized city or the unrecognized city) differed significantly in that participants were faster on trials in which they recognized neither of the two presented cities, $F(3,11)=2.98$; $p=.04$ (Table 3); a Kolmogorov-Smirnov test indicates that the means of the subjects RTs were normally distributed (all $Z$ values $>$.527). Regarding the RU trials, participants were only $156 \mathrm{msec}$ faster when choosing 


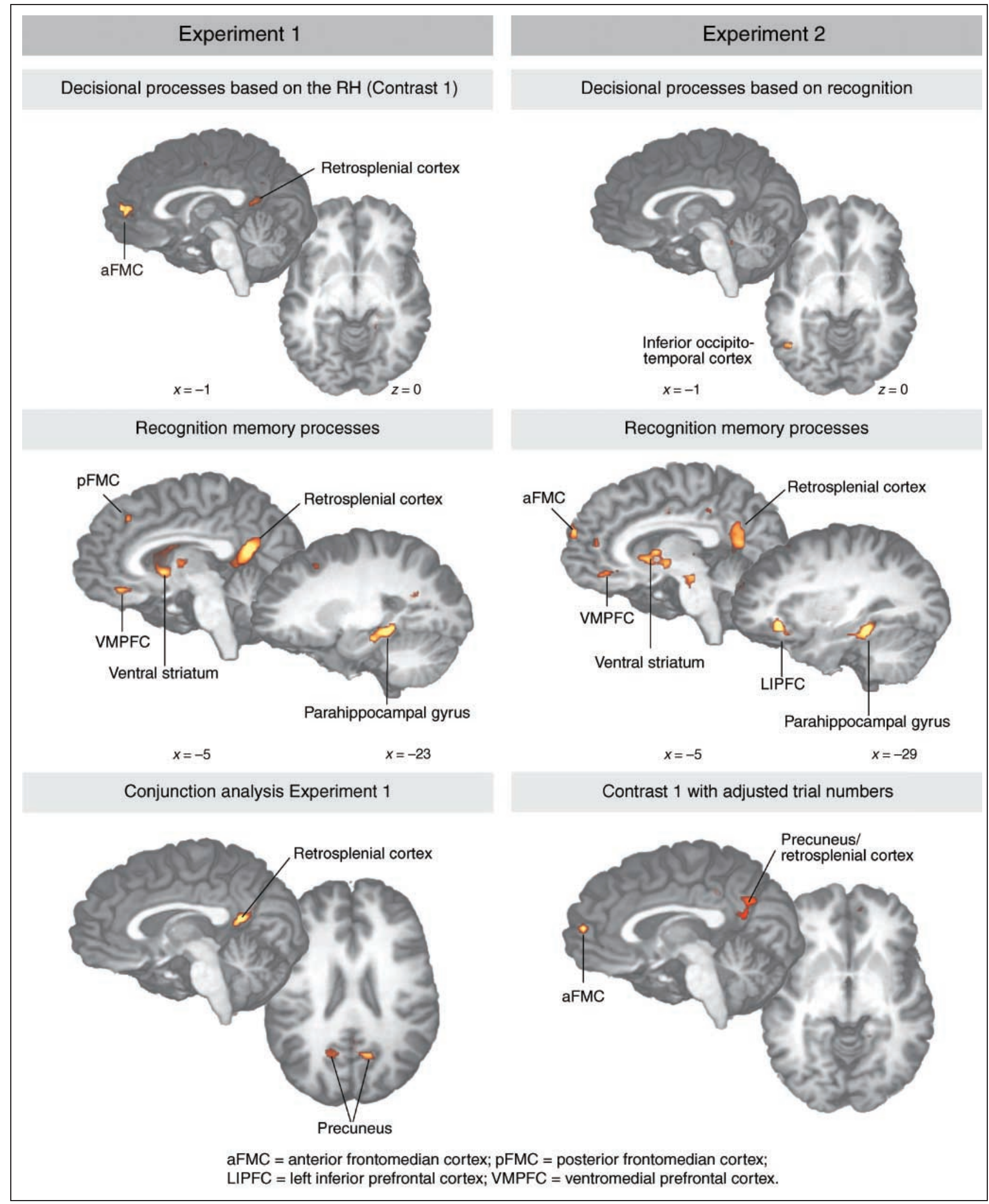

Figure 2. fMRI results of Experiments 1 and 2. Top: Results are shown for the effects of decisional processes based on the RH in Experiment 1 (contrast: RU trials in which the RH was applied $>$ RU trials in which the RH was not applied) and effects of decisional processes solely based on recognition in Experiment 2 (contrast: RU trials in which the recognized alternative was chosen $>$ RU trials in which the unrecognized alternative was chosen). Middle: The effects of recognition memory processes are shown for both experiments (contrasts: RR trials > UU trials). Bottom: left, results of the conjunction analysis of the two pictured contrasts in Experiment 1; right, result of Contrast 1 when trial numbers were adjusted. 
Table 2. Experiment 1

\begin{tabular}{lrrrr}
\hline Area & $x$ & $y$ & $z$ & $Z$ \\
\hline RH-based decisional processes & & & & \\
aFMC & -5 & 54 & 6 & 4.6 \\
Retrosplenial cortex & 4 & -48 & 15 & 3.8 \\
Medial parietal cortex & -17 & -62 & 24 & 3.9 \\
& 10 & -62 & 27 & 4.1
\end{tabular}

Table 3. Behavioral Results of Experiment 2

\begin{tabular}{lcc}
\hline Condition & $R T$ & $S E(R T)$ \\
\hline $\mathrm{RR}$ & 3114 & 69 \\
$\mathrm{UU}$ & 3015 & 86 \\
$\mathrm{RU}(\mathrm{R})$ & 3121 & 111 \\
$\mathrm{RU}(\mathrm{U})$ & 3277 & 70 \\
\hline
\end{tabular}

Reaction times (RTs) of the following conditions are shown: participants recognized both cities (RR), no city (UU), participants recognized one city and had to choose the recognized alternative $\left(\mathrm{RU}_{\mathrm{R}}\right)$ or had to choose the unrecognized alternative $\left(\mathrm{RU}_{\mathrm{U}}\right)$.

the recognized alternative as compared to choosing the unrecognized alternative, $t(13)=-1.94 ; p=.08$.

\section{MRI Results}

Generally, to control for the RT differences between the conditions, we included RT parameters for each condition as in Experiment 1. Parallel to the heuristic contrast in Experiment 1, we compared trials in which participants recognized one alternative and chose the recognized one with trials in which they recognized one alternative and chose the unrecognized one. A significantly higher hemodynamic activity was elicited within the left inferior occipitotemporal cortex and inferior frontal sulcus when participants chose the recognized city (Figure 2, Table 4).

Table 4. Experiment 2

\begin{tabular}{lrrrr}
\hline Area & $X$ & $y$ & $z$ & $Z$ \\
\hline Decisional processes based on recognition & only & & \\
Inferior occipitotemporal cortex & -44 & -59 & 0 & 3.8 \\
Inferior frontal sulcus & -44 & 36 & 9 & 3.6 \\
& & & & \\
Recognition memory processes & & & & \\
Midportion of the parahippocampal & -29 & -38 & -6 & 4.4 \\
$\quad$ gyrus & 25 & -36 & -11 & 3.6 \\
Precuneus extending into & -11 & -59 & 15 & 4.0 \\
$\quad$ retrosplenial cortex & & & & \\
VMPFC & -8 & 39 & -9 & 4.0 \\
Ventral striatum extending into the & -2 & 7 & 3 & 4.7 \\
$\quad$ caudate nucleus & & & & \\
aFMC (Brodmann's area 9) & -11 & 60 & 24 & 4.2 \\
LIPFC & -29 & 28 & -8 & 4.4 \\
\hline
\end{tabular}

VMPFC $=$ ventromedial prefrontal cortex aFMC $=$ anterior frontomedian cortex; LIPFC = left inferior prefrontal cortex.

Anatomical specification, Talairach coordinates $(x, y, z)$, and maximal $Z$ scores of significantly activated voxels are shown for each contrast calculated in Experiment 2. 
To investigate which brain areas were involved with successful recognition we contrasted RR trials with UU trials parallel to Experiment 1. Significant activation was observed bilaterally within the midportion of the parahippocampal gyrus, right precuneus extending into retrosplenial cortex, bilaterally within the ventral striatum extending into caudate nucleus, LIPFC, aFMC (Brodmann's area 9), and VMPFC (Figure 2, Table 4). A conjunction analysis between these two contrasts revealed no area to be commonly activated.

To identify overlap in activation patterns for successful recognition memory processes in Experiments 1 and 2 , we calculated a test for the logical AND (Nichols et al., 2005). Regions that revealed to be commonly activated by recognition memory processes in both experiments were bilaterally the midportion of the parahippocampal gyrus, precuneus extending into the retrosplenial cortex, ventral striatum bilaterally, and LIPFC. Activation within the MTL and medial parietal cortex have repeatedly been associated with recognition memory processes (e.g., Henson, 2005; Wagner et al., 2005), and LIPFC activation with controlled semantic processing (e.g., Gold, Balota, Kirchhoff, \& Buckner, 2005). Thus, the activation obtained in these experiments suggests that recognition memory processes are engaged both when assessing which city was larger (Experiment 1) and when assessing which city was recognized (Experiment 2).

\section{DISCUSSION}

The present study investigated the neural correlates of decisional processes that are based on the RH. Simply stated, the $\mathrm{RH}$ claims that when making a judgment about two items, a person recognizing only one of the alternatives will infer the recognized one has the higher criterion value. The effective use of the RH depends on the outcome of two processes: whether the alternatives are recognized and a judgment about the ecological rationality of the $\mathrm{RH}$, that is, whether the chain of correlations linking the (inaccessible) criterion to recognition through a mediator variable justifies following the RH. Accordingly, RH-based decisional processes are special in that they feature a judgment of ecological rationality compared to decisional processes that solely rely on recognition information.

In the following we will discuss the medial parietal activation and the frontomedian activation that was found to be specific to the application of the RH. Subsequently, we will discuss why there was no frontomedian activation when the RH was not applied.

\section{Activation within Medial Parietal Areas Reflect Recognition Processes When Decisions Follow the RH}

Generalizing from anatomical studies in monkeys, the precuneus and retrosplenial cortex are upstream to the medial temporal memory system (Kobayashi \& Amaral, 2003). Thus, medial parietal areas are directly or indirectly connected to the MTL. The retrosplenial cortex is special in that its afferent connections are dominated by MTL projections. Based on this finding, Kobayashi and Amaral (2003) suggested parietal midline structures as the major pathways through which the MTL influences cortical information processing in the service of declarative memory. This assumption is further supported by a phenomenon referred to as "retrosplenial amnesia." Damage to the retrosplenial cortex has been associated with the loss of verbal episodic memory as well as with a loss for spatial relations (Maeshima et al., 2001; Valenstein et al., 1987). Imaging studies repeatedly reported activation within parietal midline structures that extend from the retrosplenial cortex and posterior cingulate to the precuneus for episodic memory retrieval processes (Krause et al., 1999; Maguire, Frith, \& Morris, 1999; Squire et al., 1992): Activation within medial parietal areas, specifically within the precuneus, has been shown to be sensitive to successful retrieval (old/new effects; Henson, Rugg, Shallice, Josephs, \& Dolan, 1999; Fletcher et al., 1995), autobiographical memory retrieval (Addis, McIntosh, Moscovitch, Crawley, \& McAndrews, 2004; Gilboa, Winocur, Grady, Hevenor, \& Moscovitch, 2004), retrieval orientation (Dobbins, Rice, Wagner, \& Schacter, 2003), and for the subjective perception that information is old (perceived recognition; Kahn, Davachi, \& Wagner, 2004; Wheeler \& Buckner, 2003). Yet, at the same time, precuneus activation has also been found in studies of visuospatial imagery, selfprocessing operations, and consciousness (for an overview, see Cavanna \& Trimble, 2006; Naghavi \& Nyberg, 2005). Given the involvement of the precuneus in such a wide spectrum of higher order cognitive functions, the exact nature of the precuneus function has long been considered elusive, and domain-specific interpretations dominated. By reviewing current knowledge about the anatomical and cytoarchitectonic structure of the precuneus as well as functional imaging data, Cavanna and Trimble (2006) have suggested a functional subdivision within the precuneus: an anterior region that is especially involved in self-centered mental imagery strategies, and a posterior region mainly subserving successful episodic memory retrieval, irrespective of the imagery content of the retrieved information.

The precuneus activation in the present study for RHbased decisional processes occurred in the posterior part of the precuneus, specifically anterior to the parietoocciptial sulcus, and thus is suggested to be associated with successful retrieval attempts. This interpretation is in line with the assumption that the precuneus particularly responds to familiarity confidence (Yonelinas, Otten, Shaw, \& Rugg, 2005). Yonelinas et al. (2005) reported a positive correlation between posterior precuneus activation and recognition confidence, suggesting that activation within the posterior precuneus can be 
used as proxy for the overall strength of the recognition signal. Additional support for this comes from our parametric analysis linking medial posterior parietal cortex with how likely a city was recognized by our participants. Experiment 1 revealed higher activation within the aFMC and precuneus extending into the retrosplenial cortex when participants responded according to the $\mathrm{RH}$ (i.e., choosing the recognized city as larger) as compared to when they did not. The activation foci that were elicited by $\mathrm{RH}$-based decisional processes (i.e., aFMC, precuneus, and retrosplenial cortex) could be dissociated with respect to their involvement in recognition memory processes: The medial parietal areas alone were found to be activated both by the application of the $\mathrm{RH}$ and recognition. Accordingly, we suggest medial parietal cortex activation to mainly reflect recognition memory processes during RH-based decisions, whereas aFMC activation could be specific to judgmental processes about the RH's ecological rationality.

\section{Activation within the aFMC Reflects Judgments of Ecological Rationality When Decisions Follow the RH}

Based on Experiment 1, one could entertain the hypothesis that aFMC is doing nothing more than accumulating a recognition signal from medial parietal areas, implying that decisions are made strictly on the basis of recognition. This suggests that to the extent that RH-based decisional processes can adequately be explained by participants simply choosing the recognized alternative, aFMC activation should also be elicited by decisional processes based solely on recognition information. To test this hypothesis, we ran Experiment 2, but instead asked participants to choose the recognized alternative in each trial. Contrasting trials (in Experiment 2) in which participants chose the recognized alternative with trials in which they did the opposite revealed no significant aFMC activation, but instead activation within the left inferior occipitotemporal cortex. This area has repeatedly been found for lexical decisions in the visual domain (e.g., for word-pseudoword discrimination; Fiebach, Friederici, Muller, \& von Cramon, 2002). This finding led to the assumption that occipitotemporal brain areas could be conceived of as a functionally specialized word recognition region within the ventral visual pathway. Fiebach and Friederici (2002) replicated the lexical decision task in the auditory domain and again found activation within the occipitotemporal cortex for real words as compared to pseudowords, which led the authors to suggest this area to be related to modalityindependent aspects of word recognition. Accordingly, when participants (in Experiment 2) chose the recognized city, they simply opted for that word whose word representation form was stored in their lexicon.
These results further bolster the claim that the aFMC activation observed in Experiment 1 is specific to judgments of ecological rationality and not merely for the strength of the recognition signal. Recently, the aFMC has been proposed to reflect self-related processes or social-cognitive judgments (Northoff \& Bempohl, 2004; Ochsner et al., 2004; Gusnard, Akbudak, Shulman, \& Raichle, 2001). Generally, such processes can be characterized by the requirement to relate an aspect of the external world to oneself involving the ranking, scaling, and evaluating of one's own priorities and notions in relation to parameters of the external situation (Goldberg \& Podell, 1999). A feature of paradigms investigating the cerebral correlates of self-related processes is that responses cannot be scored against an absolute standard; rather, correct responses are relative and actor centered, such as what is conceived of as moral (Heekeren, Wartenburger, Schmidt, Schwintowski, \& Villringer, 2003; Moll et al., 2002; Greene, Sommerville, Nystrom, Darley, \& Cohen, 2001) or as coherent (Ferstl \& von Cramon, 2002) or as beautiful (Jacobsen, Schubotz, Hofel, \& Cramon, 2006). In the city judgment task in Experiment 1, participants had to evaluate the applicability of the $\mathrm{RH}$, which could be done by inferring a rough correlation between the (strength of) recognition and city population. Hence, judgments of the RH's ecological rationality are largely self-referential, as they depend on assessing one's own sense of recognition.

\section{Why Wasn't There aFMC Activation When the RH Was Not Followed?}

One might expect judgments of ecological rationality should be required in those trials in which the $\mathrm{RH}$ was not applied. Yet, these trials did not elicit aFMC activation. To investigate whether aFMC activation results from a relative difference in signal strength between the two sorts of trials or rather because trials in which the RH was not applied did not activate the aFMC at all, we plotted the time course of the mean percentage signal change in the aFMC. Results revealed less deactivation for trials in which the $\mathrm{RH}$ was applied as compared to those trials in which the $\mathrm{RH}$ was not applied (Figure 3). The time course of the aFMC supports the default-mode hypothesis ascribed to the medial prefrontal cortex that was put forward by Gusnard et al. (2001). The finding that the aFMC is among those brain regions showing the highest baseline metabolic activity and taskrelated activation reductions during goal-directed cognitive processing led to the assumption that the medial prefrontal cortex subserves functions that are essential to the self-concept: During attention-demanding tasks the self-referential activity of the aFMC is reduced. In the present study, activation in the aFMC was reduced more when participants failed to apply the $\mathrm{RH}$, suggesting that the suspension of the $\mathrm{RH}$ required more cognitive effort 
Figure 3. Mean percentage signal changes (MPSCs, i.e., event-related averages) with error bars for the aFMC, (right) precuneus, and retrosplenial cortex in Experiment 1: Top, MPSCs are shown for the aFMC for trials in which the RH was applied (red line) and was not applied (blue line). Middle, MPSCs in the (right) precuneus for all conditions. Bottom, MPSCs for the retrosplenial cortex (green, RR trials; black, UU trials). The stimulus presentation of the two city names, lasting for $4 \mathrm{sec}$, started at time point zero.

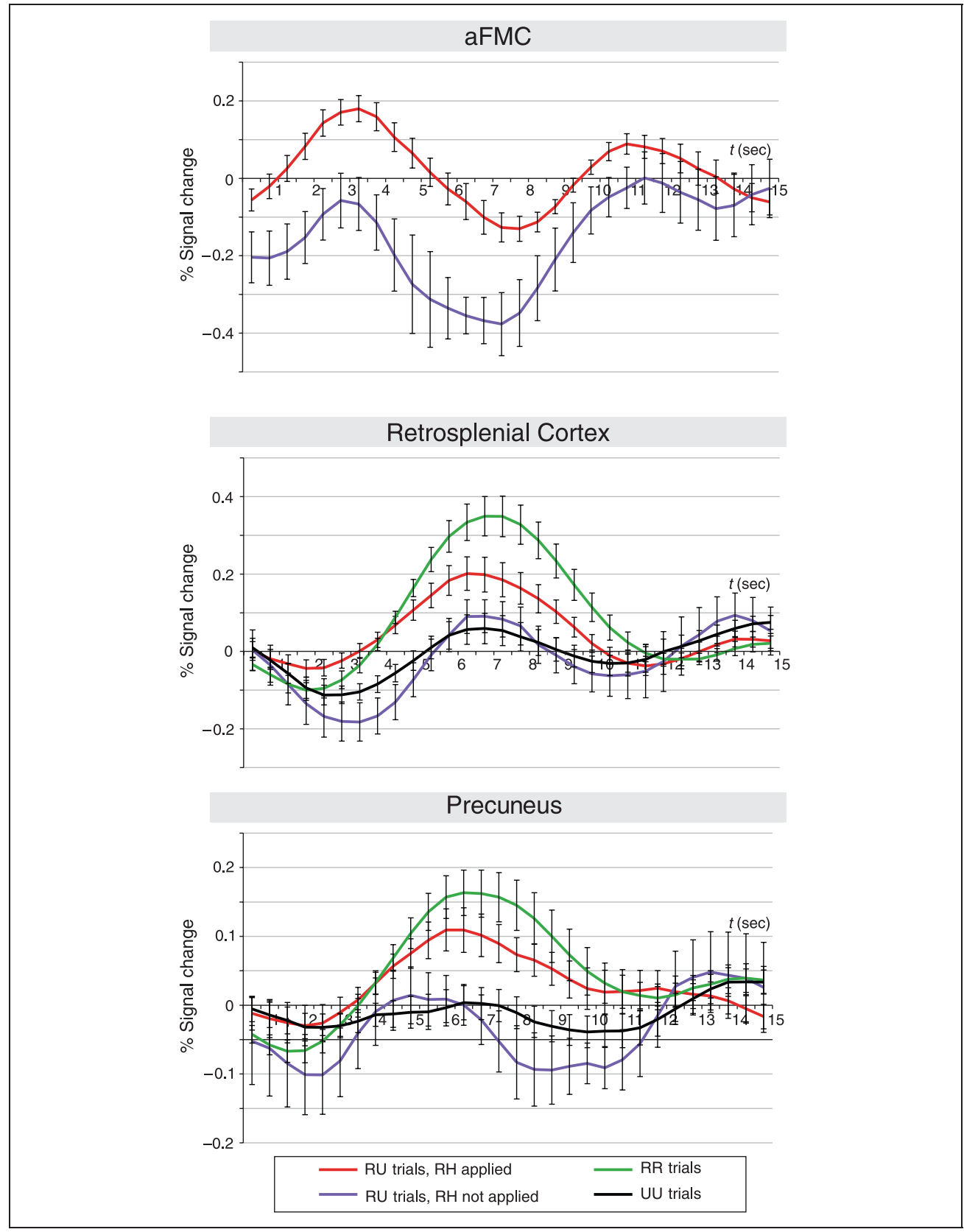

than judgments in line with the heuristic, suggesting participants follow the $\mathrm{RH}$ by default. This conclusion is consistent with the RT data: Participants in Experiment 1 took significantly longer to decide against the RH, that is, when assessing the unrecognized alternative as the larger city $(F(1,17)=30.2 ; p=.0001)$. The finding that it takes additional time to suspend the $\mathrm{RH}$ has also been reported by Pachur and Hertwig (in press).

There could be multiple reasons that would lead participants to go against the RH. For instance, a person could discount their recognition because they know that the city is small (Oppenheimer, 2003). For example, the city of Pisa in Italy is probably known by its leaning tower, so participants may infer that the recognition of
Pisa is not directly indicative of a large population size (about 86,000 inhabitants). Another possibility is that they are not only taking into account whether they recognize the city or not, but how confident they are that they recognize it. Evidence for this comes from the time course for activation in the precuneus and retrosplenial cortex when the $\mathrm{RH}$ was followed and when it was not. Figure 3 shows activation in the precuneus and retrosplenial areas for the four kinds of trials of interest in Experiment 1. Notice that the UU, $\mathrm{RU}_{(\mathrm{R})}$, and RR trials are ordered in terms of the number of alternatives recognized, in line with the suggestion of Yonelinas et al. (2005) that the precuneus reflects recognition confidence and our parametric analysis of 
recognition rates and activation in these regions. The surprising result is the paltry activation in medial parietal areas for the $\mathrm{RU}_{(\mathrm{U})}$ trials where the activation is not significantly different from the signal of UU trials, that is, when neither item was recognized [precuneus: $t(17)=$ $-0.42 ; p=.68$; retrosplenial cortex: $t(17)=-1.5$; $p=.17]$ (Figure 3), consistent with the hypothesis that they failed to follow the $\mathrm{RH}$ when recognition confidence is low.

Taken together, the present fMRI studies converge on the following conclusions: (1) Whereas RH-based decisions do depend on the strength of the recognition signal, as measured by activation in medial parietal areas, (2) the processes underlying RH-based decisions go beyond simply choosing the recognized alternative. (3) Specifically, RH-based decisional processes are distinguished by judgments about the ecological rationality of the RH that are specific to the aFMC.

\section{Acknowledgments}

The authors thank Jennifer Kittel for assistance in data collection, Ralph Hertwig for valuable considerations and productive discussions, and two anonymous reviewers for their very constructive and helpful comments on a prior version of the manuscript.

Reprint requests should be sent to Kirsten G. Volz, Max Planck Institute for Human Cognitive and Brain Sciences P.O. Box 500 355, D-04303 Leipzig, Germany, or via e-mail: volz@cbs. mpg.de, www.cbs.mpg.de.

\section{REFERENCES}

Addis, D. R., McIntosh, A. R., Moscovitch, M., Crawley, A. P., \& McAndrews, M. P. (2004). Characterizing spatial and temporal features of autobiographical memory retrieval networks: A partial least squares approach. Neuroimage, 23, 1460-1471.

Aggleton, J. P., Van, S. D., Denby, C., Dix, S., Mayes, A. R., Roberts, N., et al. (2005). Sparing of the familiarity component of recognition memory in a patient with hippocampal pathology. Neuropsychologia, 43, 1810-1823.

Aggleton, J. P., \& Shaw, C. (1996). Amnesia and recognition memory: A re-analysis of psychometric data. Neuropsychologia, 34, 51-62.

Birn, R. M., Cox, R. W., \& Bandettini, P. A. (2002). Detection versus estimation in event-related fMRI: Choosing the optimal stimulus timing. Neuroimage, 15, 252-264.

Cavanna, A. E., \& Trimble, M. R. (2006). The precuneus: A review of its functional anatomy and behavioural correlates. Brain, 129, 564-583.

Dobbins, I. G., Rice, H. J., Wagner, A. D., \& Schacter, D. L. (2003). Memory orientation success: Separable neurocognitive components underlying episodic recognition. Neuropsychologia, 41, 318-333.

Ferstl, E. C., \& Cramon, Y. D. (2002). What does the frontomedian cortex contribute to language processing: Coherence or theory of mind? Neuroimage, 17, 1599-1612.

Fiebach, C. J., \& Friederici, A. D. (2002). Neural correlates of lexicality and word frequency in the auditory lexical decision task. In A. D. Friederici \& D. Y. von Cramon (Eds.),
Annual report 2002 (pp. 28-29). Leipzig: Max Planck Institute for Human Cognitive and Brain Sciences.

Fiebach, C. J., Friederici, A. D., Muller, K., \& von Cramon, D. Y. (2002). fMRI evidence for dual routes to the mental lexicon in visual word recognition. Journal of Cognitive Neuroscience, 14, 11-23.

Fletcher, P. C., Frith, C. D., Grasby, P. M., Shallice, T., Frackowiak, R. S., \& Dolan, R. J. (1995). Brain systems for encoding and retrieval of auditory-verbal memory. An in vivo study in humans. Brain, 118, 401-416.

Forman, S. D., Cohen, J. D., Fitzgerald, M., Eddy, W. F., Mintun, M. A., \& Noll, D. C. (1995). Improved assessment of significant activation in functional magnetic resonance imaging (fMRI): Use of a cluster-size threshold. Magnetic Resonance in Medicine, 33, 636-647.

Friston, K. J., Fletcher, P., Josephs, O., Holmes, A., Rugg, M. D. \& Turner, R. (1998). Event-related fMRI: Characterizing differential responses. Neuroimage, 7, 30-40.

Friston, K. J., Frith, C. D., Turner, R., \& Frackowiak, R. S. (1995). Characterizing evoked hemodynamics with fMRI. Neuroimage, 2, 157-165.

Gilboa, A., Winocur, G., Grady, C. L., Hevenor, S. J., \& Moscovitch, M. (2004). Remembering our past: Functional neuroanatomy of recollection of recent and very remote personal events. Cerebral Cortex, 14, 1214-1225.

Gold, B. T., Balota, D. A., Kirchhoff, B. A., \& Buckner, R. L. (2005). Common and dissociable activation patterns associated with controlled semantic and phonological processing: Evidence from fMRI adaptation. Cerebral Cortex, 15, 1438-1450.

Goldberg, E., \& Podell, K. (1999). Adaptive versus veridical decision making and the frontal lobes. Consciousness and Cognition, 8, 364-377.

Goldstein, D. G., \& Gigerenzer, G. (2002). Models of ecological rationality: The recognition heuristic. Psychological Review, 109, 75-90.

Greene, J. D., Sommerville, R. B., Nystrom, L. E., Darley, J. M., \& Cohen, J. D. (2001). An fMRI investigation of emotional engagement in moral judgment. Science, 293, 2105-2108.

Gusnard, D. A., Akbudak, E., Shulman, G. L., \& Raichle, M. E. (2001). Medial prefrontal cortex and self-referential mental activity: Relation to a default mode of brain function. Proceedings of the National Academy of Sciences, U.S.A., 98, 4259-4264.

Heekeren, H. R., Wartenburger, I., Schmidt, H., Schwintowski, H. P., \& Villringer, A. (2003). An fMRI study of simple ethical decision-making. NeuroReport, 14, 1215-1219.

Henson, R. (2005). A mini-review of fMRI studies of human medial temporal lobe activity associated with recognition memory. Quarterly Journal of Experimental Psychology, Series B, Comparative and Physiological Psychology, 58, 340-360.

Henson, R. N. A., Rugg, M. D., Shallice, T., Josephs, O., \& Dolan, R. J. (1999). Recollection and familiarity in recognition memory: An event-related functional magnetic resonance imaging study. Journal of Neuroscience, 19, 3962-3972.

Jacobsen, T., Schubotz, R. I., Hofel, L., \& Cramon, D. Y. (2006). Brain correlates of aesthetic judgment of beauty. Neuroimage, 29, 276-285.

Kahn, I., Davachi, L., \& Wagner, A. D. (2004). Functionalneuroanatomic correlates of recollection: Implications for models of recognition memory. Journal of Neuroscience, 24, 4172-4180.

Kobayashi, Y., \& Amaral, D. G. (2003). Macaque monkey retrosplenial cortex: II. Cortical afferents. Journal of Comparative Neurology, 466, 48-79. 
Krause, B. J., Schmidt, D., Mottaghy, F. M., Taylor, J., Halsband, U., Herzog, H., et al. (1999). Episodic retrieval activates the precuneus irrespective of the imagery content of word pair associates. A PET study. Brain, 122, 255-263

Lohmann, G., Muller, K., Bosch, V., Mentzel, H., Hessler, S., Chen, L., et al. (2001). LIPSIA-A new software system for the evaluation of functional magnetic resonance imaging of the human brain. Computerized Medical Imaging and Graphics, 25, 449-457.

Maeshima, S., Ozaki, F., Masuo, O., Yamaga, H., Okita, R., \& Moriwaki, H. (2001). Memory impairment and spatial disorientation following a left retrosplenial lesion. Journal of Clinical Neuroscience, 8, 450-451.

Maguire, E. A., Frith, C. D., \& Morris, R. G. (1999). The functional neuroanatomy of comprehension and memory: The importance of prior knowledge. Brain, 122, 1839-1850.

Miezin, F. M., Maccotta, L., Ollinger, J. M., Petersen, S. E., \& Buckner, R. L. (2000). Characterizing the hemodynamic response: Effects of presentation rate, sampling procedure, and the possibility of ordering brain activity based on relative timing. Neuroimage, 11, 735-759.

Moll, J., de Oliveira-Souza, R., Eslinger, P. J., Bramati, I. E., Mourão-Miranda, J., Andreiuolo, P. A., et al. (2002). The neural correlates of moral sensitivity: A functional magnetic resonance imaging investigation of basic and moral emotions. The Journal of Neuroscience, 22, 2730-2736.

Naghavi, H. R., \& Nyberg, L. (2005). Common fronto-parietal activity in attention, memory, and consciousness: Shared demands on integration? Consciousness and Cognition, 14, 390-425.

Nichols, T., Brett, M., Andersson, J., Wager, T., \& Poline, J. B. (2005). Valid conjunction inference with the minimum statistic. Neuroimage, 25, 653-660.

Northoff, G., \& Bermpohl, F. (2004). Cortical midline structures and the self. Trends in Cognitive Sciences, 8, 102-107.

Norris, D. G. (2000). Reduced power multislice MDEFT imaging. Journal of Magnetic Resonance Imaging, 11, 445-451.

Ochsner, K. N., Knierim, K., Ludlow, D. H., Hanelin, J., Ramachandran, T., Glover, G., et al. (2004). Reflecting upon feelings: An fMRI study of neural systems supporting the attribution of emotion to self and other. Journal of Cognitive Neuroscience, 16, 1746-1772.

Oppenheimer, D. M. (2003). Not so fast! (and not so frugal!): Rethinking the recognition heuristic. Cognition, 90, B1-B9.

Pachur, T., \& Hertwig, R. (in press). On the psychology of the recognition heuristic: Retrieval primacy as a key determinant of its use. Journal of Experimental Psychology: Learning, Memory, and Cognition.
Pohl, R. F. (2006). Empirical tests of the recognition heuristic. Journal of Behavioral Decision Making. Ranganath, C., Yonelinas, A. P., Cohen, M. X., Dy, C. J., Tom, S. M., \& D'Esposito, M. (2003). Dissociable correlates of recollection and familiarity within the medial temporal lobes. Neuropsychologia, 42, 2-13.

Rugg, M. D., \& Yonelinas, A. P. (2003). Human recognition memory: A cognitive neuroscience perspective. Trends in Cognitive Sciences, 7, 313-319.

Squire, L. R. (1994). Declarative and non-declarative memory: Multiple brain systems supporting learning and memory. In D. L. Schacter \& E. Tulving (Eds.), Memory systems (pp. 203-231). Cambridge: MIT Press.

Squire, L. R., Ojemann, J. G., Miezin, F. M., Petersen, S. E., Videen, T. O., \& Raichle, M. E. (1992). Activation of the hippocampus in normal humans: A functional anatomical study of memory. Proceedings of the National Academy of Sciences, U.S.A., 89, 1837-1818.

Squire, L. R., Stark, C. E. L., \& Clark, R. E. (2004). The medial temporal lobe. Annual Review of Neuroscience, 27, 279-306.

Talairach, P., \& Tournoux, J. (1998). Co-planar stereotactic atlas of the buman brain. Stuttgart: Thieme.

Thirion, J. P. (1998). Image matching as a diffusion process: An analogy with Maxwell's demons. Medical Image Analysis, 2, 243-260.

Ugurbil, K., Garwood, M., Ellermann, J., Hendrich, K., Hinke, R., Hu, X. P., et al. (1993). Imaging at high magnetic fields: Initial experiences at $4 \mathrm{~T}$. Magnetic Resonance Quarterly, 9, 259-277.

Valenstein, E., Bowers, D., Verfaellie, M., Heilman, K. M., Day, A., \& Watson, R. T. (1987). Retrosplenial amnesia. Brain, 110, 1631-1646.

Wagner, A. D., Shannon, B. J., Kahn, I., \& Buckner, R. L. (2005). Parietal lobe contributions to episodic memory retrieval. Trends in Cognitive Sciences, 9, 445-453.

Wheeler, M. E., \& Buckner, R. L. (2003). Functional dissociation among components of remembering: Control, perceived oldness, and content. Journal of Neuroscience, 23, 3869-3880.

Worsley, K. J., \& Friston, K. J. (1995). Analysis of fMRI time-series revisited--again. Neuroimage, 2, 173-181.

Ward, B. D. (2000). Simultaneous inference for fMRI data. Available: afni.nimh.nih.gov/afni/doc/manual/AlphaSim [state 2006-01-06].

Yonelinas, A. P., Otten, L. J., Shaw, K. N., \& Rugg, M. D. (2005). Separating the brain regions involved in recollection and familiarity in recognition memory. The Journal of Neuroscience, 25, 3002-3008.

Yonelinas, A. P., Kroll, N. E., Quamme, J. R., Lazzara, M. M., Sauve, M. J., Widaman, K. F., et al. (2002). Effects of extensive temporal lobe damage or mild hypoxia on recollection and familiarity. Nature Neuroscience, 5 , 1236-1241. 